\title{
Error reporting from the da Vinci surgical system in robotic surgery: A Canadian multispecialty experience at a single academic centre
}

\author{
Emad Rajih, $M D^{1,2,3}$; Côme Tholomier, $M D^{1}$; Beatrice Cormier, $M D^{4,5}$; Vanessa Samouëlian, $M D^{4,5}$; \\ Thomas Warkus, MD',5; Moishe Liberman, MD'; Hugues Widmer, MD'; Jean-Baptiste Lattouf, MD'; \\ Abdullah M. Alenizi, MD'; Malek Meskawi, MD'; Roger Valdivieso, MD'; Pierre-Alain Hueber, $M D^{\prime}$; \\ Pierre I. Karakewicz, MD'; Assaad El-Hakim, MD'; Kevin C. Zorn, $M D^{1}$
}

\begin{abstract}
'Division of Urology, University of Montreal Hospital Centre (CHUM), Montreal, QC, Canada; ${ }^{2}$ Urology Department, Taibah University, Madinah, Saudi Arabia; ${ }^{3}$ Division of Robotic Urology, Department of Surgery, Hôpital du Sacré Cœur de Montréal, Montreal, QC, Canada; ${ }^{4}$ Gynecologic Oncologic Division, University of Montreal Hospital Centre (CHUM), Montreal, QC, Canada; ${ }^{5}$ Institut du Cancer, CRCHUM, Montreal, QC, Canada; ' Division of Thoracic Surgery, University of Montreal Hospital Centre (CHUM), Montreal, QC, Canada
\end{abstract}

Cite as: Can Urol Assoc J 2017;11 (5):E197-202. http://dx.doi.org/10.5489/cuaj.4116 Published online May 9, 2017

\section{Abstract}

Introduction: The goal of the study is to evaluate and report on the third-generation da Vinci surgical (Si) system malfunctions.

Methods: A total of 1228 robotic surgeries were performed between January 2012 and December 2015 at our academic centre. All cases were performed by using a single, dual console, four-arm, da Vinci Si robot system. The three specialties included urology, gynecology, and thoracic surgery. Studied outcomes included the robotic surgical error types, immediate consequences, and operative side effects. Error rate trend with time was also examined.

Results: Overall robotic malfunctions were documented on the da Vinci Si systems event log in 4.97\% (61/1228) of the cases. The most common error was related to pressure sensors in the robotic arms indicating out of limit output. This recoverable fault was noted in $2.04 \%$ (25/1228) of cases. Other errors included unrecoverable electronic communication-related in 1.06\% (13/1228) of cases, failed encoder error in $0.57 \%(7 / 1228)$, illuminator-related in $0.33 \%(4 / 1228)$, faulty switch in $0.24 \%(3 / 1228)$, battery-related failures in $0.24 \%(3 / 1228)$, and software/hardware error in $0.08 \%$ $(1 / 1228)$ of cases. Surgical delay was reported only in one patient. No conversion to either open or laparoscopic occurred secondary to robotic malfunctions. In 2015 , the incidence of robotic error rose to $1.71 \%(21 / 1228)$ from $0.81 \%(10 / 1228)$ in 2014.

Conclusions: Robotic malfunction is not infrequent in the current era of robotic surgery in various surgical subspecialties, but rarely consequential. Their seldom occurrence does not seem to affect patient safety or surgical outcome.

\section{Introduction}

Over the past decade, the da Vinci robotic system has been widely adopted by multiple surgical specialties, with notable improvement over conventional approaches with regards to blood loss and hospital stay. ${ }^{1-3}$ From a surgeon ergonomic perspective, the advantages of robotic-assistance include the Endowrist ${ }^{\circledast}$ free movement, three-dimensional magnified vision, and surgeon comfort in a seated position. ${ }^{4}$ With paucity of randomized studies, the issue of robotic safety still remains in the spotlight in the surgical scientific literature and mainstream media. ${ }^{5,6}$

The da Vinci surgical system is a computer-assisted device with three main integrated subsystems: 1) the surgeon console, which is the control centre of the system; 2) the patientside cart, including the robot with articulated mechanical arms; and 3) vision cart, which contains supporting hardware and software components, including the electrical surgical unit (ESU). The locations of robotic errors can potentially occur in all these subsystems.

Very few publications have demonstrated the reliability and safety of the initial generations of robotic systems. ${ }^{5}$ However, at least one report published in 2015 reviewed the events reported to the U.S Food and Drug Administration (FDA) between 2000 and 2012, with an alarming count of 71 deaths and 174 non-fatal injuries (conversion to open, nerve damage, reoperation, permanent damage, and visceral injury) that occurred in robotic-assisted surgeries. ${ }^{6}$ Nevertheless, this study reported on surgical injuries and complications at large that occurred during robotic surgery in different surgical specialties rather than reflecting on the incidence and consequences of specific robotic malfunctions.

Newer generations have evolved, including a more compact, dual-console da Vinci system ( $\mathrm{Si}$ and $\mathrm{Xi}$ ) and device error rates of current systems have not been studied broadly. Therefore, we sought to determine the reliability and safety of the da Vinci Si system by multiple users on a single device at a tertiary care centre. 
Rajih et al.

\section{Methods}

After institutional board approval, data were retrospectively reviewed from our institutional surgical database. The study included all patients treated surgically by the da Vinci system at the University of Montreal Hospital Centre (CHUM) from January 2012 to December 2015 (Table 1). Surgical specialties included urology, gynecologic-oncology, and thoracic surgery. All procedures were performed by one of seven surgeons on a single, dual-console, four-arm da Vinci Si robotic system. During the study period, four surgeons began their initial experience with robotics under close proctorship in 2013. All recorded errors that were identified by the da Vinci system were automatically stored by the system in a flash hard drive. Timing of the reported errors, such as during system start-up prior to anesthesia and during shut down after undocking, was identified as well. Intraoperative robotic malfunctions were also evaluated. Moreover, materials that were obtained from the single da Vinci unit computer's hard drive were further evaluated for recoverable and non-recoverable errors. Non-recoverable errors were considered as a major error and failure, since they usually mandate the need to shut down the system and replacement of the failed part if required. All cases were consecutively collected over the study period. We aim to determine the reliability and safety of the da Vinci Si system by multiple users on a single device at a tertiary care centre.

\section{Outcomes measures}

Primary measures were collected, including case abortion secondary to robotic malfunctions and conversion to either laparoscopic or open procedures. Physical injury and death that resulted from the robotic malfunction or direct contact trauma from different robotic parts were evaluated. Surgical delay in which the time required to correct robotic malfunction exceeded 10 minutes was recorded accordingly.

\section{Descriptive data analysis}

The data were created and analyzed from the databases of different surgical specialties. Robotic errors were divided into two categories: recoverable and unrecoverable strata.
Furthermore, the robotic errors were classified according to the time of error during surgery, technical system involved, and year of surgery. Descriptive statistics using frequencies and proportions of robotic error occurrence were used.

\section{Results}

Analysis of the da Vinci console hard drive matched our clinical outcomes database, with 1228 robotic procedures included. Table 1 summarizes the different surgical procedures that were performed during the study period.

The number of robotic cases performed in 2012, 2013, 2014, and 2015 were 139 (11.32\%), 310 (25.24\%), 395 $(32.17 \%)$, and $384(31.27 \%)$, respectively. There were 61 $(4.97 \%)$ recorded errors on the computer hard drive system, $46(3.75-75.4 \%$ of total errors) of which were recoverable and 15 (1.22-24.5\% of total errors) of which were non-recoverable. Errors before patient anesthesia (during the robotic setup by the dedicated robotic nursing staff) and after robotic undocking were recorded in 15 cases, 93.33\% (14/15) of which were managed without any patient consequence or surgical delay. Surgical delay leading to prolonged operative time was observed in only one case $(6.67 \%)$. This was related to battery loss, which was corrected immediately with a replacement battery before docking. There was no impact on the surgical outcome, but the operative time was increased by 45 minutes. The remaining $46(3.74 \%)$ cases occurred while patients were anesthetized between docking and undocking.

In our cohort, two cases were not completed robotically due to clinical indication, but none had to be converted or aborted because of robotic malfunction (Table 2).

The most common observed malfunction in our series robotic arm output/power limit exceeded - was provoked by arm collision ( 25 cases or $41 \%$ ), all of which were recoverable errors. In such instances, the system froze temporarily; however, surgery was quickly resumed simply by addressing the error source (e.g., by moving the resistance that was colliding with the arm). In rare occasions, we were forced to remove the instruments and replace them again. In no cases were we required to power off the system.

With regards to the observed errors, other non-arm-related faults were encountered. Detailed timing and description of robotic errors are reported in Table 3. Specific to the cam-

\begin{tabular}{|c|c|c|c|c|c|}
\hline \multicolumn{2}{|l|}{ Urology $(n=586)$} & \multicolumn{2}{|c|}{ Gynecology $(n=580)$} & \multicolumn{2}{|c|}{ Thoracic surgery (n=62) } \\
\hline Radical prostatectomy & 532 & Radical hysterectomy & 40 & Lobectomy & 4 \\
\hline Robotic partial nephrectomy & 15 & TRH BSO \& LND & 518 & Radical thymectomy & 26 \\
\hline \multirow[t]{3}{*}{ Other } & 39 & Radical trachelectomy & 5 & Mediastinal mass resection & 18 \\
\hline & & LND \& omentectomy & 10 & Nissan fundoplication & 7 \\
\hline & & Other & 7 & Other & 6 \\
\hline
\end{tabular}




\begin{tabular}{|c|c|c|}
\hline Effect & Number & Comments \\
\hline Conversion & 0 & $\begin{array}{l}\text { A case was converted due to clinical } \\
\text { indication. It was a large renal mass ( } 14 \\
\mathrm{~cm} \text { ) that was difficult to accomplish by } \\
\text { minimal invasive surgery. }\end{array}$ \\
\hline Abort & 0 & $\begin{array}{l}\text { A case was aborted due to clinical } \\
\text { indication. It was aborted due to gastric } \\
\text { tumour invading the esophagus, which } \\
\text { was unknown preoperatively. }\end{array}$ \\
\hline Delay & 1 & $\begin{array}{l}\text { Battery-related. The battery related errors } \\
\text { are recoverable, thus can be cleared from } \\
\text { the touchscreen. No need to power down } \\
\text { the system. To prevent this error from } \\
\text { recurring, the battery was replaced on the } \\
\text { same day, after the surgeries were done. }\end{array}$ \\
\hline Injury & 0 & - \\
\hline Death & 0 & - \\
\hline
\end{tabular}

era and lighting systems, we experienced four cases with an illuminator-related error. The computer system reports an error when one of the illuminator buttons is pressed for too long or if it gets stuck down after being pressed. These were recurring errors, so the whole illuminator was changed. Battery-related errors were noted in three cases in our series; one of these led to a surgical delay of 45 minutes during docking. Since engineers were on site for all cases, the batteries were replaced on the same day, immediately following all procedures.

Unrecoverable electronic communication-related errors were reported in 16 cases in our series. In fact, 12 of such events occurred during surgery. Fortunately, all are considered as just an anomaly, a sudden unexpected temporary malfunction, because they originated from a different component in the electronic system that can be corrected immediately. In such cases, it was not imperative to replace the electronic components. These types of errors are not predictable and are not preventable. At our institution, once these types of errors occur, a review of the error logs by the intuitive surgical distributer engineering team would be performed in order to replace the error components in the system after surgery.

Failed encoder error and robotic arm output/power limit exceeded error occurred in 17/25 (68.00\%) during surgery in 2013, and those were recoverable. Such errors happened when an instrument or an arm was moved in a jerky way or with collision (other arms, assistant devices, table, etc.). They are preventable with increased surgeon experience by limiting collisions and uneven movements. During these warning events, the "recover button" is simply pushed on the screen in order to continue the surgery.

\section{Discussion}

In this report, the incidence of robotic malfunction rate during surgery was $3.7 \%$, which is close to the previous- ly reported rates with the initial da Vinci generations that ranged from $0.4-2.6 \% .{ }^{4,7}$ Most importantly, these robotic malfunction errors did not translate into negative impact on patient safety or surgical outcomes. Specifically, no conversion to open surgery was observed secondary to robotic malfunction. These data also support the safety of the latest da Vinci Si systems in various surgical procedures and across different specialties.

The highest error rate occurred in $25 / 310$ cases $(8.06 \%)$ in 2013 in these series. The error rate declined significantly during the subsequent year, with a rate of $2.5 \%$ in 2014 . Most of the reported errors in $2013(17 / 25,68.00 \%)$ were physician-induced faults secondary to arm collision or jerky arm movements. This may be explained by the fact that 2013 was a learning curve period of novice surgical and nursing staff, as well as the start of thoracic surgical team.

In 2015, the incidence of robotic error rose to $5.47 \%$ (21/384 cases) compared to 2014. However, the errors related to electronic communication, not from instrument collision and therefore not linked to surgical experience. In fact, further studies are needed to investigate the occurrence of electronic communication error with time and increasing robotic use.

Andonian et al showed that the increasing use of robotic-assisted surgery has led to an increase in the number of reported device malfunctions. ${ }^{8}$ In an extensive MAUDE database review between 2000 and 2007, they reported a $0.38 \%$ estimated failure rate in 50000 robot surgeries. Similarly, Kim et al reported the device error rate among six departments during their initial experience with first-generation da Vinci system coupled with continued evaluation with the da Vinci Si system. ${ }^{9}$ While subspecialty error rates were not assessed, the observed incidence of mechanical failure was $2.39 \%(43 / 1797)$. This is comparable with the $2.6 \%$ robot error rate causing failure to complete robotic surgery during the learning curve of robotic-assisted radical prostatectomy (RARP) observed by Borden et al. ${ }^{10}$ When assessing other non-urological initial experiences, there is little evidence on the safety of robotic surgery and error rates. For example, only few case series have addressed error rates in the thoracic subspecialty. Mahieu et al did not observe any case conversions in their initial 28 robotic lobectomies. ${ }^{11}$ In a Cochrane review about robotic surgery in gynecology, robotic error rates were not reported, nor discussed. ${ }^{12}$

In our study, arm collision-related error was the most common recorded error, occurring in $2.0 \%$ of cases and representing $4.1 \%$ of total robotic faults. Such results validate those reported in the literature. ${ }^{13}$ Collision is considered a recoverable malfunction simply by moving the colliding object away. Proper ports placement and surgeon experience could decrease the incidence of arm collision-related error. ${ }^{14,15}$

In our series, only one error (battery loss) resulted in delayed surgery. Surgical delay related to robotic failure has been examined by several other studies. Kozlowski reported 
Rajih et al.

\begin{tabular}{|c|c|c|c|c|c|c|}
\hline \multirow{2}{*}{ Error (n) } & \multicolumn{2}{|c|}{ During surgery } & \multirow{2}{*}{ Year (n) } & \multicolumn{2}{|c|}{ Type(n) } & \multirow{2}{*}{ Description } \\
\hline & Yes & No & & $\mathbf{R}$ & NR & \\
\hline Illuminator-related (4) & 4 & 0 & $\begin{array}{l}2012(1) \\
2013(0) \\
2014(0) \\
2015(3)\end{array}$ & 4 & 0 & $\begin{array}{l}\text { Illuminator was left on too long and } \\
\text { illuminator brightness up and the lamp on/off } \\
\text { switches stuck. }\end{array}$ \\
\hline $\begin{array}{l}\text { Electronic communication- } \\
\text { related* }(16)\end{array}$ & 12 & 4 & $\begin{array}{l}2012(2) \\
2013(4) \\
2014(1) \\
2015(9)\end{array}$ & 1 & 15 & Due to lack of communication between boards. \\
\hline $\begin{array}{l}\text { Robotic arm output/power } \\
\text { limit exceeded }^{\dagger}(25)\end{array}$ & 25 & 0 & $\begin{array}{l}2012(2) \\
2013(11) \\
2014(6) \\
2015(6)\end{array}$ & 25 & 0 & $\begin{array}{l}\text { This type of error usually occurs when } \\
\text { instrument/arm is moved in a jerky way or } \\
\text { collides with something. }\end{array}$ \\
\hline Battery-related (3) & 1 & 2 & $\begin{array}{l}2012(0) \\
2013(2) \\
2014(0) \\
2015(1)\end{array}$ & 3 & 0 & Battery temperature was high $50-60^{\circ} \mathrm{C}$. \\
\hline Failed encoder error ${ }^{\dagger}(7)$ & 2 & 5 & $\begin{array}{l}2012(0) \\
2013(2) \\
2014(0) \\
2015(1)\end{array}$ & 7 & 0 & $\begin{array}{l}\text { This type of error usually occurs when } \\
\text { instrument/arm is moved in a jerky way or } \\
\text { collides with something. }\end{array}$ \\
\hline Software error (1) & 0 & 1 & $\begin{array}{l}2012(0) \\
2013(1) \\
2014(0) \\
2015(0)\end{array}$ & 1 & 0 & $\begin{array}{l}\text { Software/hardware failure in one electronic } \\
\text { component. It recovered immediately without } \\
\text { replacement. }\end{array}$ \\
\hline Faulty switch (3) & 0 & 3 & $\begin{array}{l}2012(0) \\
2013(1) \\
2014(2) \\
2015(0)\end{array}$ & 3 & 0 & $\begin{array}{l}\text { Type of electronic error. It is recovered } \\
\text { immediately without replacement. }\end{array}$ \\
\hline Vision related (2) & 2 & 0 & $\begin{array}{l}2012(0) \\
2013(0) \\
2014(0) \\
2015(2)\end{array}$ & 0 & 2 & \\
\hline Total (61) & 46 & 15 & $\begin{array}{l}2012(5) \\
2013(25) \\
2014(10) \\
2015(21)\end{array}$ & 44 & 17 & \\
\hline
\end{tabular}

*This error type could occur in any of the main components (patient side cart, vision cart, or surgeon console); in this record, it originated from vision cart and patient side cart; ${ }^{\dagger}$ robotic malfunctions that can be provoked by limited experience. F: recoverable fault; NR: non-recoverable fault.

malfunctions in six patients $(4.62 \%)$ in their series of 130 robotic prostatectomies. Causes included two systems joint malfunctions, one arm malfunction, one ocular monitor loss, one "power off" error, and one software conflict. ${ }^{16}$ Operative delay because of malfunction was observed in four patients.

Borden et al reported malfunction in nine (2.57\%) patients out of 350 robotic prostatectomies. ${ }^{10}$ Causes included two robotic-arm errors, two set-up joint malfunctions, one monitor loss, one metal break of console hand piece, one software error, and one power error. Delay was reported in six cases and three cases were converted to other approaches (two open and one laparoscopic).

Finally, the risk of patient injury due to robotic malfunction is rarely reported in the literature. ${ }^{17}$ Several studies reported no injuries or deaths in their series. ${ }^{9,18}$ Zorn et al reported no death or injuries to patients related to robotic failures in 725 patients, similar to results from our study. ${ }^{4}$ Furthermore, conversion to either an open or laparoscopic procedure was believed to occur more frequently with a less experienced team according to the authors. As a result, two laparoscopic and one open conversion ensued during the early period of the learning curve. The authors reported delayed operation for four patients out of 725 robotic prostatectomies $(0.55 \%)$. These were due to three optical malfunctions and one malfunction in the power-up system.

At the present time, it is probably reasonable to say that fellowship- and residency-trained robotic surgeons are significantly more comfortable with robotic surgery and perhaps are actually becoming increasingly less used to perform laparoscopic and open surgery. ${ }^{19}$ This may especially be true, as the performed robotic-assisted surgical procedures 
increased by more than $400 \%$ in the U.S and more than $300 \%$ internationally between 2007 and $2011 .{ }^{20}$ In this context of wide adoption of robotic approach, a robotic failure that mandates conversion to either open or laparoscopic surgery may raise the concern in the future that the robotic surgeon is not capable of doing the procedure using a different approach without compromising safety and outcome.

The decline in robotic malfunction provoked by the surgeon observed in this report suggests the need of a dedicated team, as well as a learning curve in order to minimize robotic malfunctions. A dedicated team ensures continuous education and training for operative room nurses, inexperienced surgeons in the robotic field, residents and fellows in order to decrease malfunction incidence and minimize the time needed to correct errors.

The availability of a technical support team during surgery at our institution sometimes helped avoid conversion to laparoscopic or open approach. In fact, where other centres do not have the engineers during surgery, case conversion or further delay could occur.

In Canada, there are 14 trained technical and clinical support representatives who support the da Vinci robotic systems. The team regularly provides live case support within the operating room and therefore are available for immediate troubleshooting support. If not in attendance, the team of representatives can be reached by telephone and if remote technical assistance is not sufficient, the technician will immediately travel to the site to help rectify issues. Furthermore, da Vinci On Site - an online remote health monitoring system - helps ensure the systems are fully functional and enables technicians to complete preventative measures before issues arise.

Generally, hospitals do not have backup robotic parts, such as illuminators or batteries, but many hospitals have backup vision system components and communication cables. Trained intuitive technicians are qualified to repair and replace robotic system parts, although vision and communication cables can be easily replaced by a qualified nurse.

Preventative maintenance is performed by intuitive trained technicians every four months. This is usually the case at most of intuitions. A detailed procedure is followed and the preventative maintenance helps ensure the safe and reliable operation of the system. Parts are replaced when required based on inspection or when recommended by system and the manufacturer. Furthermore, each time the system is powered on, the system performs a system health check and will notify the staff and technical support team if there are any issues that require attention.

Urologists at our institution are now frequent users of the robot and are well aware that robotic malfunctions are minor and almost always quickly recoverable, without serious consequences, if one has the proper technical support team around. But for other physicians (i.e., referring, consulting, etc.) and mainly for patients and family, the idea of the robot going "crazy" and causing serious injury is actually prevalent in daily practice. We feel it is important to emphasize to colleagues and patients that robotic surgery is not automated surgery; rather, it is computer-enhanced surgery. The robot does not perform any autonomous surgical maneuver, and hence there is no possibility of it "going crazy." Furthermore, malfunctions are minor and recoverable and major malfunctions or total system shut-down is exceedingly rare. Still, regular maintenance of the materials and a dedicated nursing team are recommended to minimize these recoverable and minor faults. Overall, from our experience, very few surgical delays and no patient harm were ever observed due to robot malfunction.

This study is not without limitations. Because of its retrospective design, it is more likely to suffer from selection, misclassification, or information bias. However, despite the unknown reliability and validity of the robotic error detection and recording by the system, the departmental databases that already exists had to be reviewed for all errors and delays encountered during surgery. A prospective study could discover further details regarding the reasons for errors and how they affect the flow of surgery. Nevertheless, this study presents the first review of safety in robotic surgery from the province of Quebec. Importantly, it shows that the presence of a dedicated, well-trained medical and technical team could contribute to less robotic malfunctions.

\section{Conclusion}

For all the advanced features the da Vinci system offers, it continues to be reliable. Throughout our multiuser experience, device failures did not result in any case conversion or procedure abortion. As such, a low device failure rate can be quoted when counselling patients currently undergoing robotic surgery. Most importantly, while mechanical and electronic errors can happen, they do not appear to impact surgical outcomes or patient safety. Interestingly, the presence of skilled, dedicated, experienced team contributes to declines of robotic errors.

Competing interests: The authors report no competing personal or financial interests.

Acknowledgments: The authors would like to thank surgical robotics technical team and engineers for their continuous support in maintaining the robotic database.

This paper has been peer-reviewed. 
Rajih et al.

\section{References}

1. Eichel L, Ahlering TE, Clayman RV. Robotics in urologic surgery: Risks and benefits. AUA Update Ser 2005:24:106-11

2. Skolarus TA, Zhang Y, Hollenbeck BK. Robotic surgery in urologic oncology: Gathering the evidence. Expert Rev Pharmacoecon Outcomes Res 2010;10:421-32. https://doi.org/10.1586/erp.10.46

3. Kaye DR, Mullins JK, Carter HB, et al. Robotic surgery in urological oncology: Patient care or market share? Nat Rev Urol 2015;12:55-60. https://doi.org/10.1038/nrurol.2014.339

4. Zorn KC, Gofrit ON, Orvieto MA, et al. Da Vinci robot error and failure rates: Single-institution experience on a single three-arm robot unit of more than 700 consecutive robot-assisted laparoscopic radical prostatectomies. J Endourol 2007;21:1341-4. htrps://doi.org/10.1089/end.2006.0455

5. Kaushik D, High R, Clark CJ, et al. Malfunction of the da Vinci robotic system during robotic-assisted laparoscopic prostatectomy: An international survey. J Endourol 2010;24:571-5. hittps://doi.org/10.1089/ end.2009.0489

6. Cooper MA, Ibrahim A, Lyu H, et al. Under-reporting of robotic surgery complications. J Healthc Qual 2015;37:133-8. https://doi.org/10.1111/ihq.12036

7. Lavery HJ, Thaly R, Albala, D, et al. Robotic equipment malfunction during robotic prostatectomy: A multiinstitutional study. J Endourol 2008;22:2165-8. https://doi.org/10.1089/end.2007.0407

8. Andonian $S, 0$ keke $Z, 0$ keke DA, et al. Device failures associated with patient injuries during robot-assisted laparoscopic surgeries: A comprehensive review of FDA MAUDE database. Can I Urol 2008; 15:3912-6.

9. Kim WT, Ham WS, Jeong W, et al. Failure and malfunction of da Vinci surgical systems during various robotic surgeries: Experience from six departments at a single institute. Urology 2009;74:1234-7. https://doi.org/10.1016/i.urology.2009.05.071

10. Borden LS Jr, Kozlowski PM, Porter CR, et al. Mechanical failure rate of da Vinci robotic system. Can J Urol 2007; 14:3499-501.

11. Mahieu J, Rinieri P, Bubenheim M, et al. Robot-assisted thoracoscopic surgery vs. video-assisted thoracoscopic surgery for lung lobectomy: Can a robotic approach improve short-term outcomes and operative safety? Thorac Cardiovasc 2016;64:354-62.
12. Liu H, Lawrie TA, Lu D, et al. Robotic-assisted surgery in gynecology. Cochrane Database Syst Rev 2014;10;12:CD011422.

13. Chen $\mathrm{CC}, \mathrm{Ou} Y \mathrm{YC}$, Yang $\mathrm{CK}$, et al. Malfunction of the da Vinci robotic system in urology. Int I Urol 2012;19:736-40. https://doi.org/10.1111/j.1442-2042.2012.03010.x

14. Parnell BA, Midia EC, Fielding JR, et al. Relationship between race and abdominal anatomy: Effect on robotic port placement. Female Pelvic Med Reconstr Surg 2013;19:165-8. https://doi.org/10.1097/ SPV.0b013e318288ad6d

15. Sun LW, Van Meer F, Schmid J, et al. Advanced da Vinci surgical system simulator for surgeon training and operation planning. Int J Med Robotics 2007;3:245-51. https://doi.org/10.1002/rss.139

16. Kozlowski PM, Porter CR, Corman JM. Mechanical failure rate of da Vinci robotic system: Implantations for preoperative patient counselling. J Urol 2006;175:372-3

17. Lucas SM, Pattison EA, Sundaram CP. Global robotic experience and the type of surgical system impact the types of robotic malfunctions and their clinical consequences: An FDA MAUDE review. BJU Int 2011;109:1222-7. https://doi.org/10.1111/i.1464-410X.2011.10692.x

18. Nayyar R, Gupta NP. Critical appraisal of technical problems with robotic urological surgery. BJU Int 2010;105:1710-3. https://doi.org/10.1111/i.1464-410X.2009.09039.x

19. Wright JD, Tergas Al, Hou JY, et al. Effect of regional hospital competition and hospital financial status on the use of robotic-assisted surgery. JAMA Surg 2016;151:612-20. https://doi.org/10.1001/ jamasurg. 2015.5508

20. Carreyrou J. Surgical robot examined in injuries. Wall Street Journal 2010, May 4.

Correspondence: Dr. Kevin C. Zorn, Division of Urology, University of Montreal Hospital Centre (CHUM), Montreal, QC, Canada; Zorn.chumurology@gmail.com 